\title{
EPR Study of the Thermal Decomposition of Transannular Peroxide of Anthracene
}

\author{
Lubomír Lapčík Jr. ${ }^{1}$, Barbora Lapčíková ${ }^{1}$, Andrej Staško ${ }^{2}$ \\ ${ }^{1}$ Centre of Polymer Systems, Faculty of Technology, Tomas Bata University, Zlín, Czech Republic \\ ${ }^{2}$ Slovak Technical University Bratislava, Faculty of Chemical \& Food Technology, Bratislava, Slovak Republic \\ E-mail: lapcik@ft.utb.cz, lapcikova@ft.utb.cz, andrej.stasko@stuba.sk \\ Received April 7, 2011; revised May 15, 2011; accepted May 23, 2011
}

\begin{abstract}
Thermal decomposition of transannular peroxide of anthracene (POA) (or 9,10-epidioxido anthracene) was studied by means of electron paramagnetic resonance spectroscopy (EPR) in the solid as well as in the liquid phases. Decomposition process proceeds via cleavage of the O-O bridge of the POA molecule, generating thus an alcoxy intermediate radical. Its concentration increases to a certain equilibrium stage during the time scale of the experiment. EPR spectra in the solid state were of the singlet type at the temperatures over $350 \mathrm{~K}$, a doublet like anisotropic spectra were measured at the room temperature, both having g-value 2.0033. EPR spectrum from POA decomposed in benzene indicates four protons with higher $\left(2 a_{H}=0.305 \mathrm{mT}, 2 a_{H}=\right.$ $0.335 \mathrm{mT})$ and four protons with a lower $\left(2 a_{H}=0.075 \mathrm{mT}, 2 a_{H}=0.105 \mathrm{mT}\right)$ splitting constants, corresponding well the radical expected after cleavage of $\mathrm{O}-\mathrm{O}$ bridge.
\end{abstract}

Keywords: Endoperoxide, Anthracene, Free Radicals, EPR, Decomposition, Kinetics

\section{Introduction}

UV light irradiated anthracene and its derivatives in degassed solutions give anthracene dimers, while in the presence of oxygen they form endoperoxides [1,2]. As found by quantum chemical molecular orbital calculations based on Woodward-Hoffman orbital symmetry conservation principle application [3], endoperoxides of anthracene production is catalysed by transition metals atoms. These thermally and photo chemically reactive substances (depending on the reaction conditions) are decomposed to the original hydrocarbon and simultaneously liberate singlet oxygen $\left({ }^{1} \mathrm{O}_{2}\right)$, or decompose by the disruption of the $\mathrm{O}-\mathrm{O}$ bonds, thus producing highly reactive biradical, or finally by the transformation into the other types of peroxides [4]. For instance, for anthracene-9,10-endoperoxide (POA) it has been found, that its photochemical excitation to $S_{n}$, with $n \geq 2$ leads to cycloreversion, producing anthracene and ${ }^{1} \mathrm{O}_{2}$, whereas excitation to the $S_{1}$ state would initially cause homolytic $\mathrm{O}-\mathrm{O}$ cleavage, eventually resulting in a diepoxide rearrangement product $[5,6]$.

Decomposition via disrupting of the O-O bridge can be used for an effective crosslinking of some industrially important polymers, producing thus highly crosslinked matrices, e.g. for the optical information storage [7]. In contrary to the latter mentioned polymer matrix crosslinking reactions, depending on the reaction conditions, a vigorous degradation of polysaccharide hyaluronic acid by singlet oxygen liberated from POA was reported earlier [8]. The possibility of endoperoxides to release these reactive species by unimolecular decomposition makes them interesting candidates for in vivo site-specific oxidative targeting with singlet oxygen at the present time [6,9]. The complex oxygen photosensitization in the presence of water soluble anthracene-1sulphonate was studied by means of EPR spin trapping method [10]. It was found, that the anthracene-1-sulphonate in the presence of oxygen is converted to quinone and oxygen is activated to superoxide radical intermediate. Consecutively, the latter superoxide radicals are transformed into the highly reactive hydroxyl radicals.

In the paper presented thermal decomposition of transannular peroxide of anthracene (or anthracene 9,10endoperoxide, or 9,10-epidioxido-anthracene) is studied by means of electron paramagnetic resonance spectroscopy (EPR) both in the solid as well as in the liquid phase. 


\section{Experimental}

\subsection{Materials}

Transannular peroxide of anthracene was synthesized using photosensitized reaction with methylene blue as a photo sensitizer in chloroform by the method described earlier by Foot et al. [2,11]. Final product was then purified using column chromatography (Florosil, Fluka, Switzerland) and then several times re-crystallized in vacuum. All chemicals used during synthesis were of analytical grade purity. Final colourless crystals were identified and characterized by IR spectroscopy [11] characteristic absorption bands at 1170, 950, 880, 846, and $745 \mathrm{~cm}^{-1}$ wavenumbers as shown in Figure 1, UV VIS spectroscopy [12] and GC-MS [13].

\subsection{Methods}

Infra red spectra (IR) were recorded on computer controlled Perkin Elmer 983 spectrometer (USA).

Electron paramagnetic resonance spectra were measured at room temperature on Bruker 200E SRC spectrometer controlled by Aspect 2000 computer (Bruker, Germany). All EPR spectra were simulated using standard simulation software EPRSRC (Bruker, Germany).

\section{Results and Discussion}

In Figure 2 are shown EPR spectra of POA thermal decomposition product as observed in the solid sample at

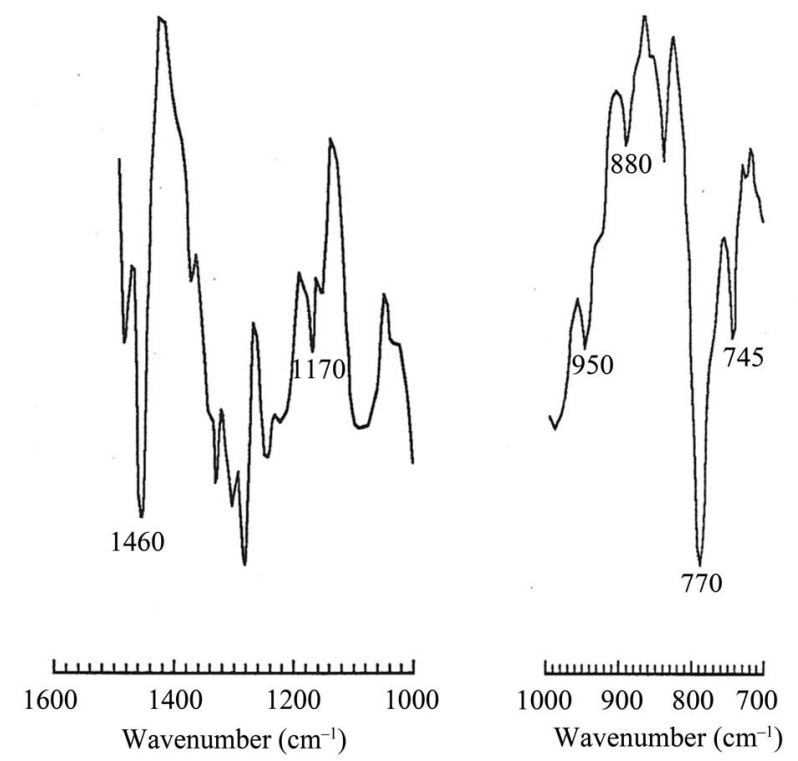

Figure 1. IR spectrum with the labelled characteristic absorption bands of POA measured in the form of thin film prepared by evaporation of solvent $\left(\mathrm{CDCl}_{3}\right)$ on $\mathrm{KBr}$ lens.

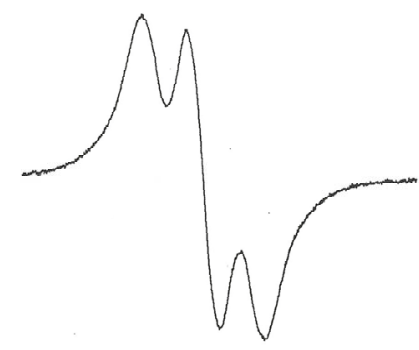

(a)

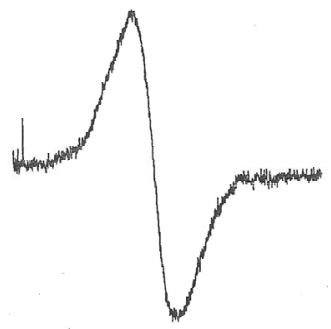

(b)
Figure 2. EPR spectra of POA measured in the solid sample at temperature: (a) $300 \mathrm{~K}$; (b) $350 \mathrm{~K}(S W=5.0 \mathrm{mT}, g=$ 2.0033).

two different temperatures. Doublet anisotropic EPR spectrum measured at $300 \mathrm{~K}$ was vanishing with the increasing temperature, while at about $350 \mathrm{~K}$ the singlet spectrum was observed. Probably, the hyperfine anisotropic structure of the EPR spectrum at lower temperature was possible to follow due to the polycrystalline character of the studied sample. As the temperature was increased to the temperature close to the melting point (values given in the literature are ranging from $120^{\circ} \mathrm{C}$ up to $160^{\circ} \mathrm{C}$ (Ref. [4]) the slightly anisotropic character disappears and only purely isotropic singlet EPR spectrum was observed. For both, the doublet as well as the singlet spectrum the same $g$-factor was observed $(g=2.00330)$, suggesting the same kind of the intermediate radical. The time dependence of the intensity of the EPR line measured at $273 \mathrm{~K}$ is shown in Figure 3. Here, the intensity of the signal is increasing with the time suggesting increasing radical concentration in the system. After several minutes, it is reaching its time independent plateau, suggesting probably equilibrium radical concentration. The same dependencies were observed at $393 \mathrm{~K}, 413 \mathrm{~K}$ as well as at $433 \mathrm{~K}$. At the temperature of $433 \mathrm{~K}$, the

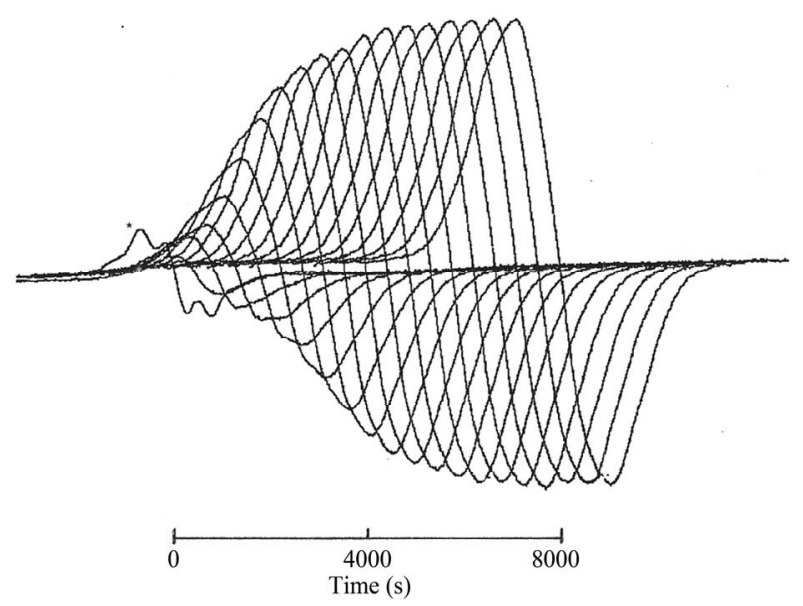

Figure 3. Time dependence of EPR spectra evolution measured at $373 \mathrm{~K}$ ( "spectrum measured at room temperature). 
intensity of the EPR spectrum after reaching its maximum decreases. Most probably, the POA is starting to decompose at this temperature. This phenomenon is more evident from Figure 4, where the kinetic curves of the time dependencies of the EPR line intensities measured at the different temperatures are shown. The initial slope is decreasing at the lower temperature. This indicates that the rate constant of the radical generation is decreasing.

Formal first order kinetic rate constants of these processes are given in the Table 1. It is evident, that with the increasing temperature the production of the radical intermediates is more effective.

Because of the fact, that the singlet EPR spectra cannot be unambiguously assigned to a defined structure, one can expect more exact structural information about the radical surrounding from the hyperfine splitting of the EPR spectra which can be observed in the solution. For this reason, we have studied the thermal decomposition of POA also in the liquid phase.

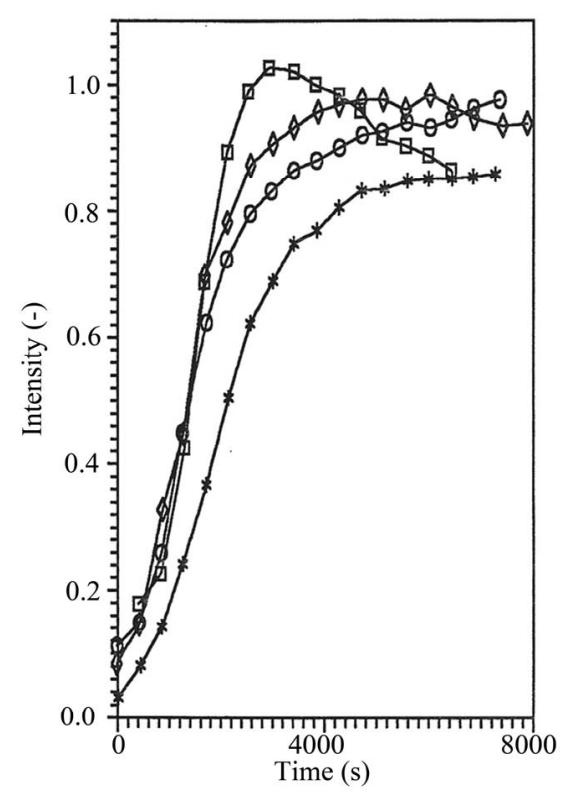

Figure 4. Time dependencies of the radical concentrations observed during thermal decomposition of POA at: star-373 K; Empty circle-393 K; Empty diamond-413 $\mathrm{K}$; Empty square-433 K.

Table 1. Obtained values of the first order kinetic mechanism rate constants of $\mathrm{POA}$ thermal decomposition as calculated for data shown in Figure 4.

\begin{tabular}{ccc}
\hline Temperature $(\mathrm{K})$ & $10^{4} \times k_{1}(1 / \mathrm{s})$ & Corr. Coeff. \\
\hline 373 & 3.32 & 0.984 \\
393 & 5.30 & 0.987 \\
413 & 6.32 & 0.981 \\
433 & 6.85 & 0.944 \\
\hline
\end{tabular}

Figure 5 shows experimental and simulated spectra of POA in benzene as observed at $300 \mathrm{~K}$. It was simulated with four higher $\left(2 a_{H}=0.305 \mathrm{mT}, 2 a_{H}=0.335 \mathrm{mT}\right)$ and four lower $\left(2 a_{H}=0.075 \mathrm{mT}, 2 a_{H}=0.105 \mathrm{mT}\right)$ splitting constants. This corresponds well to the two groups (every one with approximately four equivalent protons in $\mathrm{xx}$ and yy positions) own to POA structure.

To obtain a better fit between the experimental and simulated EPR spectrum, also a contribution singlet spectrum depicted in Figure 2(b) had to be considered by the simulation. Similar spectra were observed also at higher temperatures in different solvents (e.g. toluene, xylene, chloroform).

\section{Conclusions}

The analysis of EPR spectra shown in Figure 5 monitored during the thermal decomposition of POA suggests formation of the following radicals:

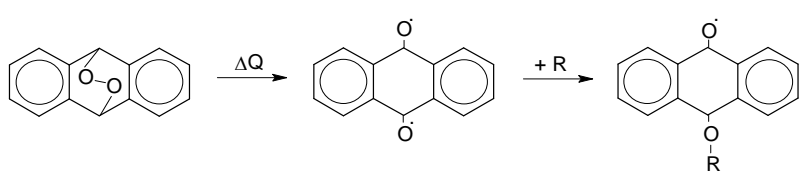

where $\mathrm{R}$ is a radical capable to react with oxygen centred radical from anthracene. $\mathrm{R}^{\bullet}$ may originate from the solvent molecules or from some another external sources. The scheme is in accord with earlier published results by Cowell and Pitts Jr. [14] assuming endoperoxide formation with consecutive radical reactions. Our theoretical investigations [3] implied the role of transition metal by the formation of antracene endoperoxide. Lazar et al. [7] experimentally confirmed formation of alkoxy radicals during thermal POA decomposition, and, furthermore, analogous EPR spectra were found investigating similar systems [15].

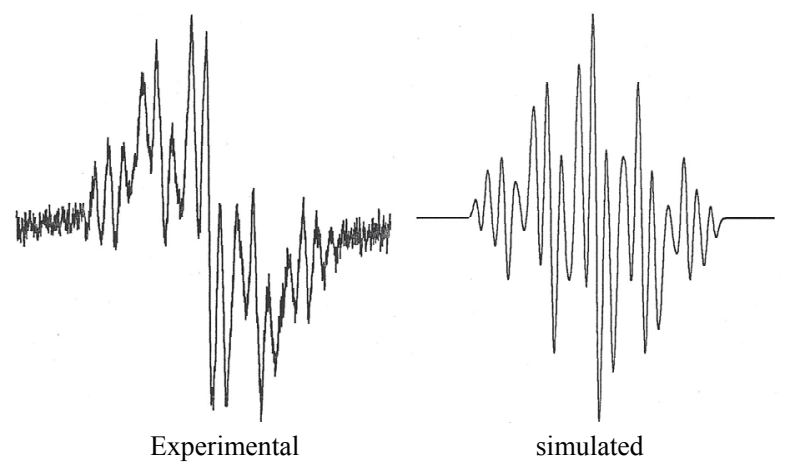

Figure 5. Experimental and simulated EPR spectra of POA dissolved in benzene during thermal treatment at $300 \mathrm{~K}, g$ $=2.0034$, simulated as $2 a_{H}=0.075 \mathrm{mT}, 2 a_{H}=0.105 \mathrm{mT}, 2 a_{H}$ $=0.305 \mathrm{mT}, 2 a_{H}=0.335 \mathrm{mT}, p p=0.045 \mathrm{mT}$ with the $g$-factor of 2.0034, $S W=2.5 \mathrm{mT}$. ( $a_{H}$ and $p p$ are splitting constant and peak-to-peak width expressed in $\mathrm{mT}$ ). 


\section{Acknowledgements}

This article was created with support of Operational Program Research and Development for Innovations co-funded by the European Regional Development Fund (ERDF) and national budget of Czech Republic within the framework of the Centre of Polymer Systems project (reg. number CZ. 1.05/2.1.00/03. 0111). This work was also supported by the Scientific Grant Agency of the Slovak Republic (Project VEGA/1/0018/09).

\section{References}

[1] J. S. K. Wan, R. N. McCormic, E. J. Baum, J. N. Pitts Jr., "The Roles of Molecular Structure and Environment in the Reactivity of Excited States," Journal of the American Chemical Society, Vol. 87, No. 20, 1965, pp. 4409-4414. doi:10.1021/ja00948a001

[2] C. S. Foote and S. Wexler, "Singlet Oxygen: A probable inter Mediate in Photosensitized Autoxidations," Journal of the American Chemical Society, Vol. 86, No. 18, 1964, pp. 3880-3881. doi:10.1021/ja01072a061

[3] M. Čeppan, L. Lapčík, M. Liška and P. Pelikán, "Molecular Orbital Study of Anthracene Photooxidation," European Polymer Journal, Vol. 16, No. 7, 1980, pp. 607-609. doi:10.1016/0014-3057(80)90096-8

[4] L. Lapčík Jr., L. Lapčík, D. Bakoš and V. Kellö, "Transannular Peroxides of Anthracene and Its Derivatives. Preparation, Properties, and Uses," Chemicke Listy, Vol. 84, No. 6, 1990, pp. 582-605.

[5] R. Schmidt, K. Schaffner, W. Trost and H. D. Brauer, "Wavelength-Dependent and Dual Photochemistry of the Endoperoxides of Anthracene and 9,10-Dimethylanthracene," Journal of Physical Chemistry, Vol. 88, No. 5, 1984, pp. 956-958. doi:10.1021/j150649a022

[6] H. Fidder, A. Lauer, W. Freyer, B. Koeppe and K. Heyne, "Photochemistry of Anthracene-9,10-endoperoxide," Journal of Physical Chemistry A, Vol. 113, No. 22, 2009, pp. 6289-6296. doi:10.1021/jp901073s

[7] S. Markuš, M. Lazár, L. Lapčík, G. Č́ík and L. Kompauer, CS Patent No. 172837, 1978, pp. 1-10.
[8] L. Lapčík Jr. and J. Schurz, "Photochemical Degradation of Hyaluronic Acid by Singlet Oxygen," Colloid and Polymer Science, Vol. 269, No. 6, 1991, pp. 633-635. doi:10.1021/jp901073s

[9] Y. Nagaoka, K. Otsu, F. Okada, K. Sato, Y. Ohba, N. Kotani and J. Fujii, "Specific Inactivation of Cysteine Protease-Type Cathepsin by Singlet Oxygen Generated from Naphthalene Endoperoxides," Biochemical and Biophysical Research Communications, Vol. 331, No. 1, 2005, pp. 215-223. doi:10.1016/j.bbrc.2005.03.146

[10] A. Staško, A. Blažková, V. Brezová, M. Breza, L. Lapčík and L. Lapčík Jr., "Oxygen Photosensitization in the Presence of Sodium Anthracene-1-sulfonate," Journal of Photochemistry and Photobiology A: Chemistry, Vol. 76, No. 3, 1993, pp. 159-165. doi:10.1016/1010-6030(93)80132-S

[11] C. S. Foote, S. Wexler, W. Ando and R. Higgins, "Chemistry of Singlet Oxygen. IV. Oxygenations with Hypochlorite-Hydrogen Peroxide," Journal of the American Chemical Society, Vol. 90, No. 4, 1968, pp. 975-981. doi: $10.1021 / \mathrm{ja} 01006 \mathrm{a} 023$

[12] I. Gillet, "Ultraviolet Absorption Spectra of the PhotoOxides of Anthracene and Naphthalene," Bulletin de la Societe Chimique de France, Vol. 17, 1950, pp. 11351141.

[13] K. Lonsdale, E. Nave and J. F. Stephens, "X-Ray Studies of a Single Crystal Chemical Reaction Photo Oxide of anthracene to (Anthraquinone, Anthrone)," Philosophical Transactions of the Royal Society of London Series A-Mathematical and Physical Sciences, Vol. 261, No. 1114, 1966, pp. 1-31. doi:10.1098/rsta.1966.0056

[14] G. W. Cowell and J. N. Pitts Jr., "Photochemical Studies in Rigid Matrixes. II. A Study of the Photochemical Reactivity of Anthracene in Polystyrene and the Development of an O-Nitrobenzaldehyde Actinometer in Poly(Methyl Methacrylate)," Journal of the American Chemical Society, Vol. 90, No. 5, 1968, pp. 1106-1110. doi:10.1021/ja01007a002

[15] "CRC Handbook of Spectra from Quinones and Quinols," CRC Press, Boca Raton, 1971. 